\title{
Regenerative and LoRa Based Trooper Monitoring System for Armed Forces
}

\author{
Hema R ${ }^{\mathrm{a}, 1}$, Dathathreya $\mathrm{P}^{\mathrm{a}}$, Athitya $\mathrm{V}{ }^{\mathrm{a}}$, Anumitha B ${ }^{\mathrm{a}}$ \\ ${ }^{a}$ Department of Electronics and Communication Engineering, Easwari \\ Engineering College, Chennai, TN, India
}

\begin{abstract}
Communication between soldier at border line is crucial. Existing system used for communication between soldiers at border line in military consumes a lot of power. The greatest difficulties in Indian armed forces operation is the Soldiers are not able to do transmission of messages with headquarters base station controller in case of emergency or when needed any help. Also, the current status and location of the soldiers cannot be detected with this system. The proposed methodology gives us Long Range (LoRa) based medical supervision and emplacement trailing and tracking system for soldiers. This type of advanced design can be mounted on the soldier's shoe to ensure their safety. In case of death of the soldier, the controller intimates to the camp office control along with soldier's location. The proposed system includes sensors, GPS, and transmission modules, as well as miniaturized wearable physiological equipment. Hence, it is possible to implement a low-cost mechanism to provide needed help in the battlefield.
\end{abstract}

Keywords: LoRaWAN, Piezoelectric Sensor, Wireless Sensor Network, Power generation

\section{Introduction}

The army administrator is not able to send any medical auxiliary to provide aid in case of poor health condition of soldiers. The existing method also does not monitor the health condition of the soldiers which is more important at war time. Thus, we move to some advanced technology which focuses on the safety of soldiers and also helps the army [1]. To overcome the disadvantages mentioned above we propose an advanced prototype design in order to provide safety for soldiers. Our proposed methodology uses piezoelectric sensor and communication using Lora. It is essential for the army headquarters controller station to know the emplacement as well as the medical condition of each individual armed force when they cross the allotted boundary. The portable remote soldier unit consists of micro controller, GPS, temperature sensor and heart beat sensor which monitor the health condition and location of soldiers [2]. If there present any abnormal condition the controller will intimate about the health parameters along with live location with the help of LORA communication network to the control room administrator along with a buzzer alert. This will be very useful for the control room to take needed action in critical situation [3].

${ }^{1}$ Hema. R, Department of Electronics and Communication Engineering, Easwari Engineering College, Chennai, TN, India; Email: hemaprabakar18@gmail.com. 
Each soldier's shoe will have a unique ID which already contains all medical records of the soldiers. This helps the medical auxiliary to provide suitable medical treatment based on soldier's health condition [4]. It also has a regenerative charging unit to power the shoes by using piezoelectric, while walking

\section{LoRaWAN}

Long Rang is abbreviated as LoRa which is widely used in wireless communication where transmission of signal takes place at low power. LoRa is a least short power broad area network technology. The working principal of LoRa is a SSMA technique which is derived from from the CSMA technique. Here CSMA means chirp spread spectrum modulation and SSMA means spread spectrum modulation method. LoRa facilitates extensive coverage of transmission and communication of signals at economically least power consumption. Other technologies and protocols such as Long-Range Wide Area Network which is abbreviated as LoRa WAN comprise the upper layers whereas LoRa technology comprises physical layer of the overall LoRa architecture. During transmission or communication of signals, values between 0.3 kbps and $27 \mathrm{kbps}$ is the baud rates which can be accomplished by the usage of LoRa technology. The one of the factors which determines this baud rate is spreading factor. Its area of coverage is broad area and LoRa transmits data at lower data rate. SemTech is a company that owns a proprietary radio modulation technology named LoRa.LoRa comprises of physical layer in LoRa protocol stack. The operation of LoRa takes place in the unlicensed ISM band. This provides an advantage which is the user does not require any license for transmission of data using the LoRa technology [5].

In India, the original frequency range of LoRa is 868-867 Megahertz. This might vary from one national region to another national region; moreover, operation of LoRa takes place over ISM band. It is very economical to be used by armed forces as it consumes rudimentary power for transmission of signals and for communication purpose at a low data rate and at higher coverage area [6] .This helps the individual armed forces to avoid the usage of cellular network which not only consumes a lot of power for transmission but also the rate of drainage of stored charge in battery is more compared to LoRa network at the cost of sacrificing higher data rate which is absent in our proposed methodology as we used LoRa WAN. Its coverage area as mentioned is in range of kilometers [7]. Transmission is bidirectional in nature with transmission and reception of signals that are done simultaneously between armed force section and the base station section .Its extensible range of transmission makes it suitable to be used at armed forces and military land based industry .It uses gateway nodes which acts as a intermediary nodes for transmission at very long distance. Signal to Noise ratio for transmission of signal is very low compared to the mobile cellular network [8]. It enables transmission and communication of signals at very low data rate covering a very large coverage area. Experimental setup: 

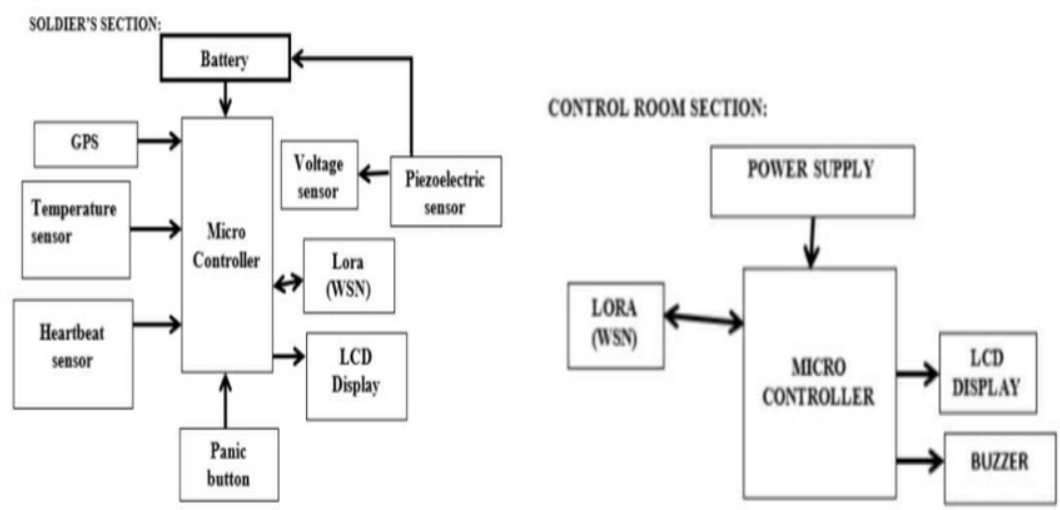

Figure 1. Block diagram of soldier section and control room section

As shown in Figure 1, Piezoelectric sensor unit is utilized by the rechargeable battery for charging purpose and this unit works on the principle of piezoelectric sensor. It generates electric voltage upon the movement of soldier during walking that is setup beneath the soldier shoe. The electric voltage generated varies upon two factors. First factor is duration for which the soldier is on movement that is total time period for which the soldier is walking. Second factor is the amount of pressure that the soldier is exerting on the setup beneath the shoe. The electric voltage generated is directly to the aforementioned two factors. Voltage sensor display that voltage generated by the piezoelectric setup. The voltage generated from piezoelectric is supplied to a rechargeable battery. The entire setup is supplied using rechargeable battery. Microcontrollers is used for processing, controlling all the signals from the different sensor of the setup. It also generates signal for enabling the LCD display and LoRa node for communication with the receiver station. Heartbeat sensor used to measure the pulse rate of the wearer and in case of any threatening abnormalities found in the values of pulse rate, it will enable the microcontroller to trigger the Lora WAN to send a alert message to headquarters situated at the base station section. Temperature sensor is utilized to find out the body temperature of the wearer, here the soldier or armed forces individual represents the wearer. PS unit of the soldier section is used by the controller room section which is also known as the headquarters situated at base station is used for tracking the live emplacement (location) of the wearer

At life threatening situation, the wearer at border line can press the panic button and enables the LoRaWAN to send a signal using microcontroller. Thereafter, the transmitted signal is received by the Base station and this will in turn trigger the buzzer located at the base station and alert the base station. Soldiers or the wearers' temperature is abnormal from the standard threshold then a alert message is sent to the receiver station. On the soldier section, a Liquid Crystal Display unit is used to show the wearer's heartbeat, battery voltage range, and temperature. LCDs perform the same functions as CRTs. Two separate LCD are used for this entire setup one on soldier section and another one is mounted on receiver section. In critical situation at border line, the wearer can trigger a panic button which activates or enable the buzzer at the receiver station. When the soldier station's panic button is pressed, the buzzer using LoRa is automatically activated. 


\section{Result and discussion}

Our proposed methodology consists of health monitoring setup which monitors the health conditions of the wearer and the entire setup is power by a rechargeable battery which is connected to piezoelectric sensor. Voltage generated by piezoelectric sensor depends upon two factors duration for which the wearer is walking and depends upon the weight that the wearer (soldier) exerts on the piezoelectric sensor as this sensor is placed beneath the foot of the wearer so that the entire body weight is exerted. Voltage generated by piezoelectric sensor which is subjected to pressure is measured by using multimeter as shown in Figure 2 and the soldier section and receiver section as shown in Figure 3.

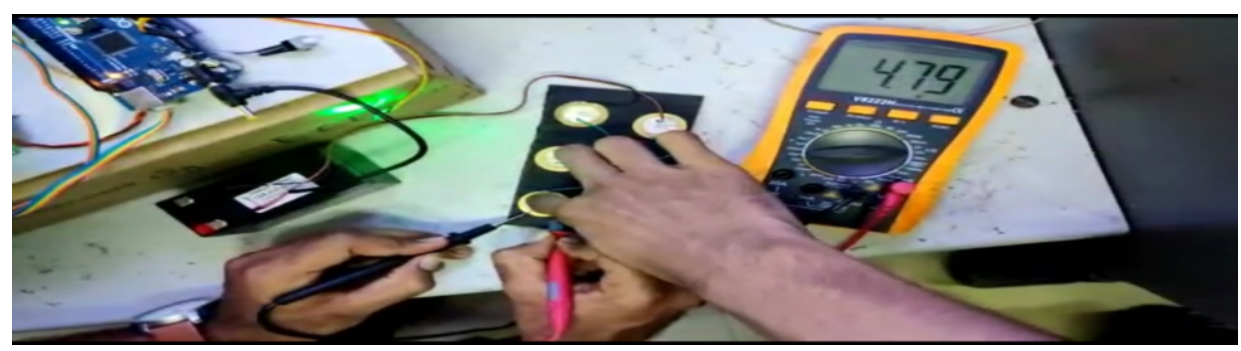

Figure 2. measurement of voltage generated at piezoelectric setup using multimeter.

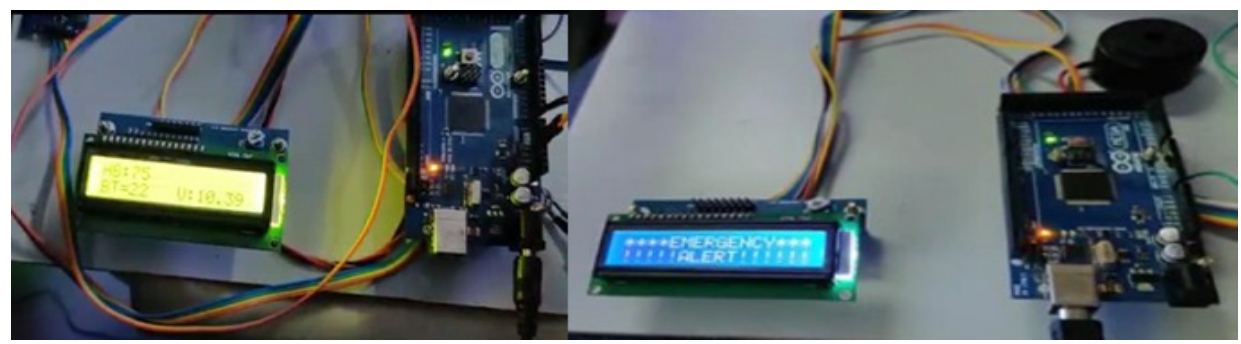

Figure 3. Soldier section and Receiver Section

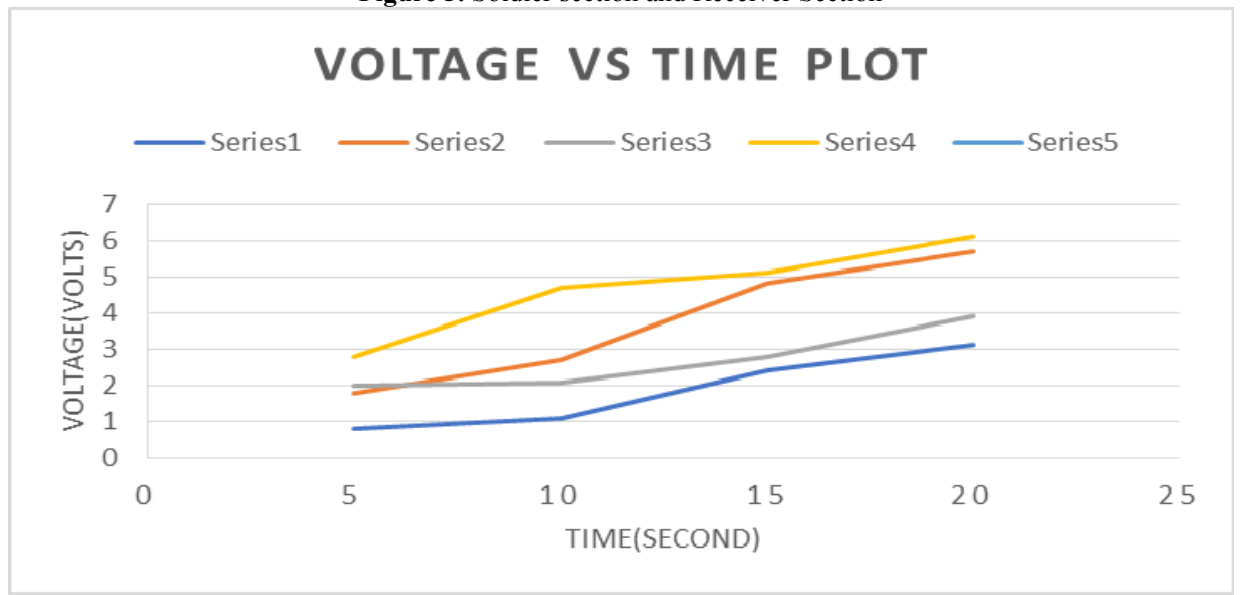

Figure 4. Voltage vs time plot (Note:series1-45kg,series2-50kg,series3-55kg,series4-60kg) 


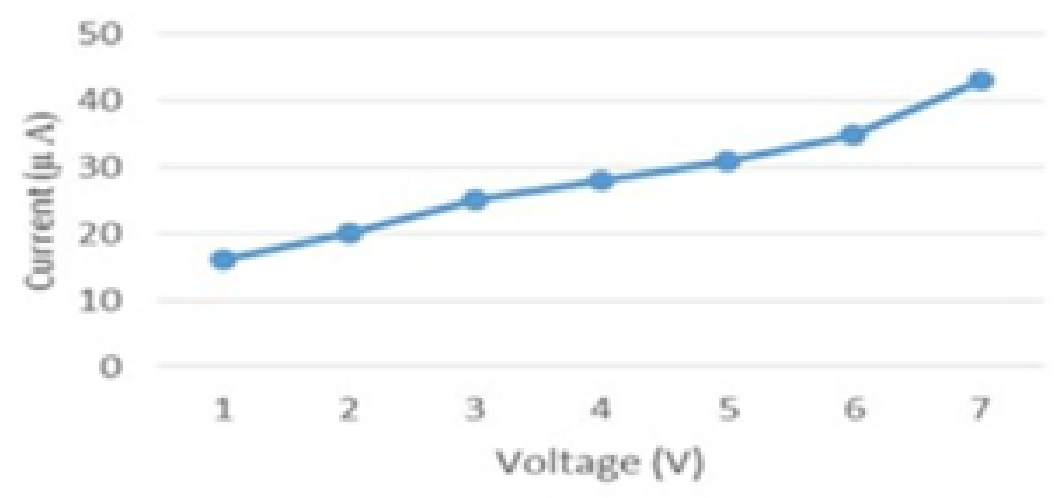

Figure 5. VI characteristics

Figure 4 represents Voltage with respect to time plot and figure 5 represents VI characteristics measured.

\section{Conclusion}

Our proposed methodology consists of cheap, economical setup wearer by the soldier for medical supervising of that particular soldier health condition who is located at border line from a place is located at the base station headquarters which represents the controller room using the LoRa technology and wireless sensor networks. Our proposed methodology avoids the wastage of the power generated .Low Signal to noise ratio is thus obtained by using this module at the same time enabling a long range transmission and communication of signal .This avoids the usage of the cellular network which transmits data at higher data rate .Our proposed methodology uses low power to transmits data signal or message at low data rate for large distance .Health monitoring consists of supervising body temperature ,heartbeat of the individual who wears this equipment .GPS unit is used to find out the emplacement of the wearer . This makes a great change in the armed forces domain by making the setup economical and cost effective which can be used for a long run as the battery is rechargeable. This is used to reduce the fatalities that happen at the border line due to lack of communication at life threatening situation of soldier to the base station.

\section{References}

[1] Zourmand, A., Sheng, N. W., Hing, A. L. K., \& Rehman, M. A. (2019). Human counting and indoor positioning system using WiFi technology. In Proceedings - 2018 IEEE International Conference on Automatic Control and Intelligent Systems, I2CACIS 2018 (pp. 142-147). Institute of Electrical and Electronics Engineers Inc. https://doi.org/10.1109/I2CACIS.2018.8603690

[2] Choi, W., Chang, Y. S., Jung, Y., \& Song, J. (2018). Low-power LORa signal-based outdoor positioning using fingerprint algorithm. ISPRS International Journal of Geo-Information, 7(11). https://doi.org/10.3390/ijgi7110440

[3] Sinha, R. S., Wei, Y., \& Hwang, S. H. (2017, March 1). A survey on LPWA technology: LoRa and NBIoT. ICT Express. Korean Institute of Communications Information Sciences. https://doi.org/10.1016/ j.icte.2017.03.004

[4] LoRa Alliance. (2015). White paper: A technical overview of LoRa and LoRaWAN. LoRaWAN-What Is It?, (November). 
[5] N. Sornin M. Luis T. Eirich T. Kramp and O. Hersent, Lo-rawan specification, LoRa Alliance Technical Report, 2015

[6] C. Jain, G. V. S. Sashank, V. N and S. Markkandan, "Low-cost BLE based Indoor Localization using RSSI Fingerprinting and Machine Learning," Sixth International Conference on Wireless Communications, Signal Processing and Networking (WiSPNET), 2021, pp. 363-367, https://doi.org/10.1109/WiSPNET51692.2021.9419388

[7] Manuel, M. P., \& Daimi, K. (2021). Implementing cryptography in LoRa based communication devices for unmanned ground vehicle applications. SN Applied Sciences, 3(4). https://doi.org/10.1007/s42452021-04377-y

[8] Ganesh Babu R., Elangovan K., Maurya S., Karthika P. (2021) Multimedia Security and Privacy on Real-Time Behavioral Monitoring in Machine Learning IoT Application Using Big Data Analytics. In: Kumar R., Sharma R., Pattnaik P.K. (eds) Multimedia Technologies in the Internet of Things Environment. Studies in Big Data, vol 79. Springer, Singapore. https://doi.org/10.1007/978-981-157965-3 9 